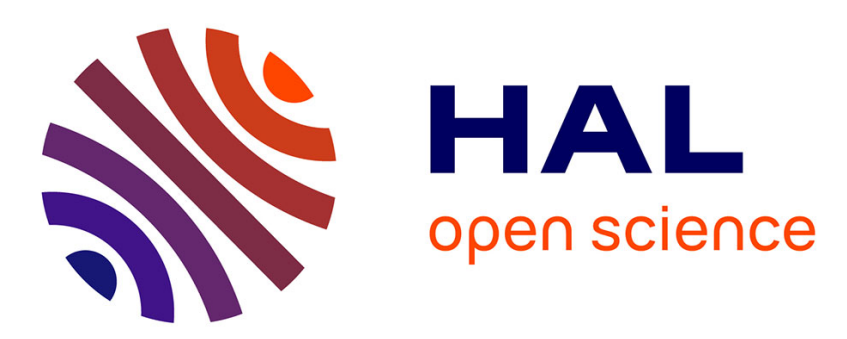

\title{
HOT ELECTRON DEVICES FOR MILLIMETRE AND SUBMILLIMETRE APPLICATIONS
}

\author{
H. Rees
}

\section{To cite this version:}

H. Rees. HOT ELECTRON DEVICES FOR MILLIMETRE AND SUBMILLIMETRE APPLICATIONS. Journal de Physique Colloques, 1981, 42 (C7), pp.C7-157-C7-170. 10.1051/jphyscol:1981718 . jpa-00221654

\section{HAL Id: jpa-00221654 https://hal.science/jpa-00221654}

Submitted on 1 Jan 1981

HAL is a multi-disciplinary open access archive for the deposit and dissemination of scientific research documents, whether they are published or not. The documents may come from teaching and research institutions in France or abroad, or from public or private research centers.
L'archive ouverte pluridisciplinaire HAL, est destinée au dépôt et à la diffusion de documents scientifiques de niveau recherche, publiés ou non, émanant des établissements d'enseignement et de recherche français ou étrangers, des laboratoires publics ou privés. 
JOURNAL DE PHYSIQUE

Colloque C7, supplément au $n^{\circ} 10$, Tome 42 , octobre 1981

page $\mathrm{C} 7-157$

HOT ELECTRON DEVICES FOR MILLIMETRE AND SUBMILLIMETRE APPLICATIONS

H.D. Rees

Royal Signals and Radar Establishment, St Andrews Road, Great Malvern, Wores WR14 3PS, United Kingdom

\begin{abstract}
Résumé: - L'importance des effets transitoires des électrons chauds dans les dispositifs semi-conducteurs augmentent avec la fréquence et, pour les ondes millimëtriques, les effets ont une grande influence sur leurs modes et leurs caractéristiques. Une étude de ces effets aide le développement des dispositifs. Les effets transitoires sont très importants pour les diodes à transfert d'électrons. Leurs modes se déforment à hautes fréquences et les meilleurs pour les sources millimëtriques sont reconnus. Pour les autres sources et les mélangeurs, les effets transitoires ont moins d'importance, mais au-delà de $100 \mathrm{GHz}$, ils ont une influence significative sur le comportement des dispositifs.

Abstract. - The importance of hot electron relaxation effects in semiconductor devices increases with frequency and, for the millimetre band, the transient processes have a major effect on their modes and terminal characteristics. A study of these effects provides a useful guide for practical device development. Relaxation effects are particularly strong in transferredelectron sources. The modes distort at high frequency and those most suitable for millimetre band generators are identified. In other sources and mixers relaxation effects are less dominant, but they have a significant influence on device philosophy above $100 \mathrm{GHz}$.
\end{abstract}

1. Introduction. - The highest frequencies of the electromagnetic spectrum offering al1-weather atmospheric propagation distances of order $1 \mathrm{~km} 1 \mathrm{ie}$ in the 70 to $400 \mathrm{GHz}$ range and consequently there is interest in the mm band for high resolution radar and radiometers. Attention has concentrated on the windows around 90 and $140 \mathrm{GHz}$, which have the best transmission in clear air, but most of the band could have applications given components of good performance and reasonable cost. Range limitations restrict the required radiated powers to levels fairly readily obtained from solid state sources at lower microwave frequencies, and these devices are versatile, compact and rugged and operate at convenient voltages. Extending their frequency is obviously an attractive idea and transmitter and local oscillator sources and diode mixers are the most urgently needed components.

To a limited extent the dimensions and doping densities of a device can be scaled for a different frequency, but the scaling laws tend to fail around the mm band. The most fundamental causes of this breakdown are hot electron relaxation effects which have characteristic distance and time scales comparable with the lengths and response times of the devices themselves. In contrast, momentum relaxation rates for unheated electrons are faster and relatively insignificant below $1 \mathrm{THz}$ except for 
cryogenically cooled devices. A study of hot electron effects is central to mm band device research because it clarifies the fundamental limiting phenomena and points out directions for improving efficiency and impedance. The latter is of major importance for minimising losses due to contact and circuit resistance,both of which become more serious with increasing frequency, and for simplifying the matching to circuits.

2. Hot Electron Relaxation Effects. - Energy relaxation times for hot electrons are typically about $1 p s$ and consequently relaxation effects will influence most band semiconductor devices in which the electrons are strongly heated. Strong heating obviously occurs in power sources such as the IMPATT and its derivatives, transferred-electron oscillators and some other sources, as yet undeveloped, which use emission from hot electrons in inversion layer states or cyclotron orbits. An apparent exception is the Schottky barrier diode mixer in which, according to the accepted models, the electron distribution function is fairly close to thermal. However at frequencies above about $100 \mathrm{GHz}$, the edge of the depletion layer can move at speeds comparable with those of thermal electrons and this opens the possibility for electrons to be heated during the reverse voltage excursion.

Hot electron relaxation effects have been studied far more for transferred-electron devices than for any other, largely because the processes associated with electron transfer can have time constants up to about 10ps, making them significant even at frequencies around $20 \mathrm{GHz}$. Power generation has been obtained for both $\mathrm{GaAs}(1)$ and InP(2) devices near $100 \mathrm{GHz}$, at which frequency relaxation effects will exert a dominant influence. This topic is of direct practical importance as well as an interesting study in hot electron physics and therefore a section of this paper deals exclusively with these effects. For other devices, relaxation phenomena are only marginally significant below $100 \mathrm{GHz}$, but will become progressively influential at higher frequencies. The character of the subject changes to include a range of effects at the onset of their significance, and for these an outline study is the first requirement. The devices considered here include the IMPATT and related transit-time oscillators, Schottky barrier mixers and more speculative possibilities including solid state analogs of vacuum tubes.

3. Modes of Transferred-electron Devices. - The basic mechanism is the same for all the semiconductors known to exhibit a strong negative resistance due to electron transfer. For the low mass, central valley electrons the scattering by polar optical phonons becomes less effective with increasing energy and, in high fields, this leads to polar runaway followed by transfer to higher mass satellite valleys. So, although materials will vary in terms of efficiency and frequency limits, the device modes will be the same and a survey may be made using any reasonable model. The present mode study includes device simulations based on an extensively used GaAs 
model (3), but both strong and weak intervalley scattering rates have been used to assess the effects of this rather uncertain parameter. ${ }^{(4)}$ Apart from this, differences between semiconductors should reflect the obvious increase of efficiency with peak to valley ratio and the increase of speed with electron scattering rate as considered by Ridley. ${ }^{(5)}$

The most obvious device concept is a bulk mode oscillator and inclusion of electron relaxation effects in calculations leads to estimates of the limiting frequency of about $100 \mathrm{GHz}$ for GaAs, with higher figures for more polar semiconductors. However, no real contacts can establish the presumed spatial uniformity and the true frequency limits reflect relaxation processes associated with instabilities. By considering the general character of the transferred-electron effect, three device modes with potential for $\mathrm{mm}$ band sources can be identified. Figure 1 schematically indicates a

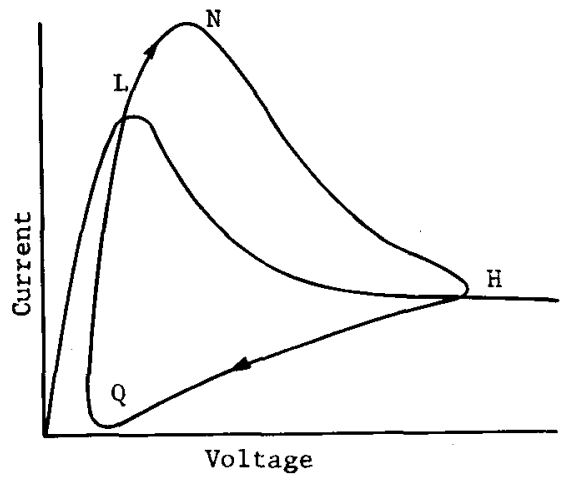

Fig. 1: Current-vo1tage characteristic

current-voltage trajectory against a scaled velocity-field curve. Ideally a device switches from a low voltage, high current state, L, to a high voltage, lower current state, $\mathrm{H}$, but the efficiency is reduced by the time spent in transitional phases, $N$, where a high field instability nucleates, and $Q$, where it is quenched. The essence of mode analysis is to quantify this figure.

An accumulation layer is the instability capable of fastest growth so its properties largely determine frequency limits. To point out the significant physics, figure 2 indicates the electric field and carrier density profiles for a device with an $\mathrm{n}^{+}$ cathode corresponding to the four phases of figure 1. If quenching is complete, the electric field on the anode side cycles between low and high values and, for a long enough device, the favourable efficiency of a bulk mode could be achieved. Complete quenching includes rethermalisation of the electrons as well as dispersal of excess charge and maintenance of the overlength mode depends on this cooling process which is slow enough to inhibit mm band oscillation. (6) 


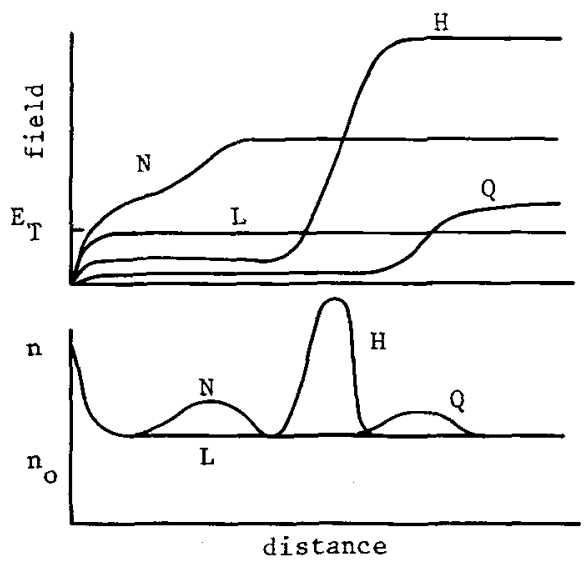

Fig. 2: Electric field and electron density in transferred-electron device

However, the frequency degradation is waveform dependent and speed is increased by adding a second harmonic phased so as to reduce the power at the fundamental. The logical extension of this is to run the device as a second harmonic generator (SHG)! This mode offers increased power and impedance because the active length can be increased to around $10 \mu \mathrm{m}$. Figure 3 , summarising a range of simulations for

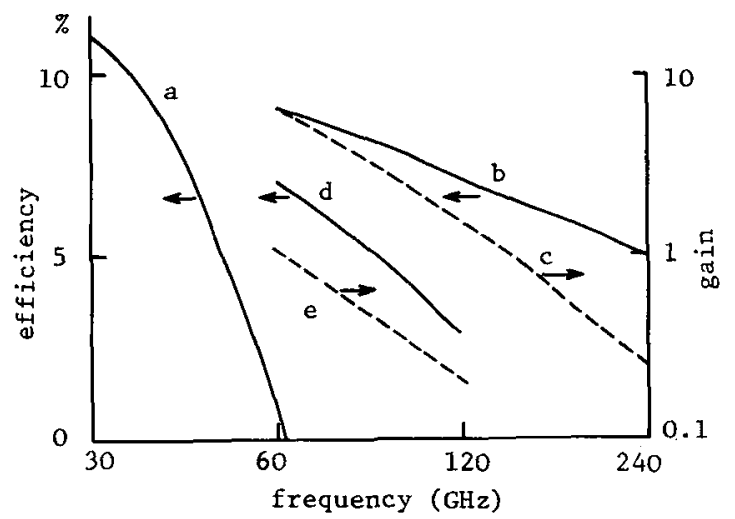

Fig. 3: Efficiency and first to second harmonic gain for $S H G$

$7 \mathrm{~mm}$ 1ong GaAs devices with $\mathrm{n}$ from 4 to $8 \times 10^{15} \mathrm{~cm}^{-3}$ compares the efficiencies of fundamental oscillators with SHG. Curves a and $b$ show the dc to rf conversion for a fundamental oscillator and a SHG respectively. Curve $c$ shows the ratio of second to first harmonic power, the rapid fall of gain with frequency being the important feature. Curves $\mathrm{d}$ and $\mathrm{e}$ correspond to $\mathrm{b}$ and $\mathrm{c}$, for a model where the intervalley scattering is 16 times weaker, the frequency limit being lowered by a factor of 2 . The picture is simplified in omitting effects of length and doping density, but the frequency improvement in a SHG is clear. The price paid in a practical power source is the need for a fundamental input signal (although this may be useful for phase stabilisation) and for a circuit matched at two harmonics, which is an advance on existing circuit design for mm band sources. 
The other two high frequency modes are obtained in shorter devices, but the cathode contacts are different. Both can be viewed as transit modes, although the bandwidth for a given length is quite large. For a device with an $\mathrm{n}^{+}$cathode the field and electron density profiles are still described qualitatively by figure 2, but for a short device the high field region is confined to the zone close to the anode. The behaviour of this mode at high frequencies is quite straightforward. Over the short distance from the cathode to the high field zone, the electron motion resembles ballistic motion $(7)$, the velocity can considerably exceed the standard peak drift velocity and the operating current is correspondingly increased. Intervalley transfer occurs quite sharply, the accumulation region is dense and is only partially dispersed during the quench phase. Speed advantages obtain because the highelectric field in the anode region increases the electron response rates and hot electrons are swept out rather than need to rethermalise. However the narrow high field zone decreases the source impedance of the oscillator and the electric field can easily approach destructive levels.

To overcome the low impendance and destructive field trends while preserving efficiency, a different cathode characteristic is needed. Current saturating and 2-zone cathodes have given good performance at lower frequencies ${ }^{(8)}$ and simulations indicate that the former, at least, maintains its advantages to the band.

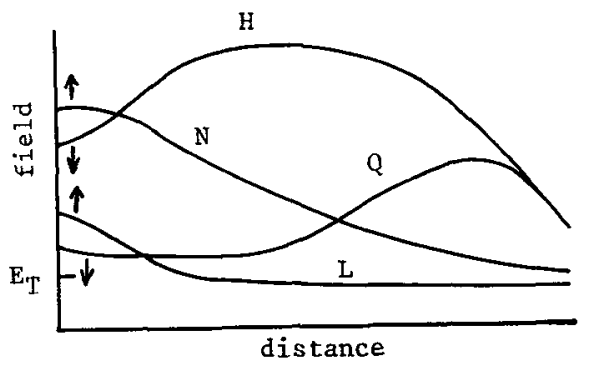

\author{
Fig. 4: Electric field in \\ device with current \\ saturating cathode \\ Arrows indicate \\ increasing or \\ decreasing cathode \\ field
}

Figure 4 shows the field profiles in a device. The oscillation has phases corresponding to those for an $\mathrm{n}^{+}$cathode, but the cathode current is purely displacement rather than purely conductive. There is partial charge depletion, which spreads out the high field region and reduces the current density. Without going into the long and complex subject of fabricating non-ohmic cathodes, an inference from the simulations is that transit oscillators with current saturating, and possibly other non-ohmic cathodes, are alternative mm band power sources.

Figure 5 summarises the efficiency-frequency characteristics derived from a series of simulations. The effects of the various device parameters are straightforward and need little comment. Curves a to $c$ are for $\mathrm{n}^{+}$cathodes. A property of these devices is that the efficiency rises with $n$ up to a level dependent on frequency and active length, which for $\mathrm{mm}$ band devices is about $10^{16} \mathrm{~cm}^{-3}$. The three curves 


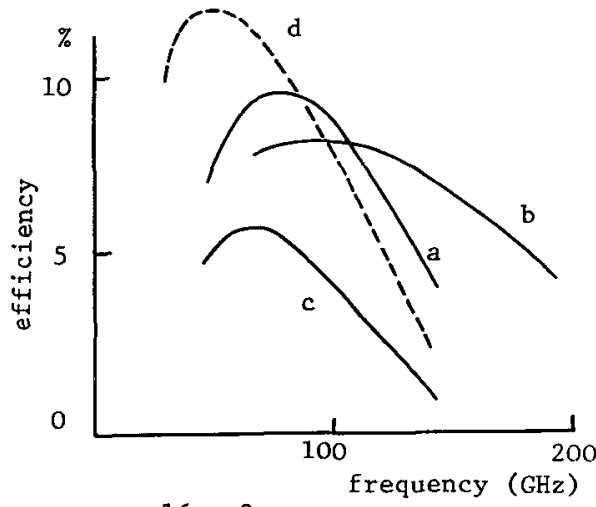

Fig. 5: Oscillator efficiency characteristics

are for $\mathrm{n}=10^{16} \mathrm{~cm}^{-3}$, curve a for a length of $2 \mu \mathrm{m}$ and $\mathrm{b}$, illustrating a weak transit length effect, for $1 \mathrm{\mu m}$. As might be expected, weaker intervalley scattering reduces the frequency limit and curve $c$, for the same structural parameters as a, but for a scattering rate reduced by 16 , illustrates the reduced frequency limit. Considering the large difference in scattering rate, the change of device characteristics is quite moderate. For current saturating cathodes, the efficiency remains high for lower values of $n$ and curve $d$ illustrates a characteristic for $b=2 \mu \mathrm{m}$. and $\mathrm{n}=5 \times 10^{15} \mathrm{~cm}^{-3}$. The optimum dc current is also lower, typically by a factor of 3 compared with an $\mathrm{n}^{+}$cathode.

For all three device modes the upper frequencies are similar, roughly 100 to $200 \mathrm{GHz}$ for GaAs depending on the strength of intervalley scattering (a figure towards the upper end of the range being supported by the high avalanche field). Under idealised conditions the efficiency should be maintained to fairly near the frequency limit, but practical realisation becomes difficult due to a combination of falling impedance, increasing current density, electric field strengths rising towards destructive levels and need for more critical circuit control. The devices differ significantly in these respects and these differences are the real theoretical guide to practical development. The rms voltage of a device is a figure of merit for power and impedance, and the quality factor, $Q$, influences contact resistance and circuit losses and bandwidth, a low $Q$ being favoured. Contact losses and thermal resistance also rise with current density. Table 1 roughly summarises the GaAs simulations for a representative frequency of $100 \mathrm{GHz}$, the dc to $x$ efficiency being roughly $7 \%$ for each device.

\begin{tabular}{|l|c|c|c|c|}
\hline & $\begin{array}{c}\text { Length } \\
(\mu \mathrm{m})\end{array}$ & $\mathrm{v}_{\mathrm{rms}}$ & $\mathrm{Q}$ & $\mathrm{A} \cdot \mathrm{cm}^{-2}$ \\
\hline SHG & 7 & 5 & 3 & $10^{4}$ \\
$\frac{\text { Transit Osc. }}{\mathrm{n}^{+} \text {cathode }}$ & 2 & 2.5 & 2 & $4 \times 10^{4}$ \\
$\begin{array}{l}\text { Current sat. } \\
\text { cathode }\end{array}$ & 2 & 2.5 & 8 & $10^{4}$ \\
\hline
\end{tabular}


It is informative to compare the numbers for a silicon IMPATT at a similar frequency. Recent good results $(9,10)$ give similar efficiencies, values for $J$ from 5 to $10 \times 10^{4}$ A. $\mathrm{cm}^{-2}$, and assuming a peak to peak $\mathrm{rf}$ voltage equal to the diode breakdown voltage, $\mathrm{v}_{\text {rms }}$ of about $5 \mathrm{~V}$. A reasonable $Q$ factor for an IMPATT is 8 . Compared with an IMPATT, the active layer thickness for a GaAs transit TEO is about 4 times greater, but $\mathrm{V}_{\text {rms }}$ and power-impedance product are lower. For InP, the threshold field is increased by a factor of 3 and this could allow proportionately higher voltages and currents. For an $\mathrm{n}^{+}$cathode device a much lower improvement would have to be accepted to keep the peak electric fields below a destructive level, but for current saturating cathodes, maximum fields would still be low. InP also offers a higher peak to valley ratio and higher speed ${ }^{(5)}$ and in this light the encouraging results from InP oscillators with metal-n cathodes is understandable ${ }^{(2)}$. However, there is probably a lot of scope for cathode engineering to give better power levels.

The SHG offers higher peak power and impedance, a representative $v_{\text {rms }}$ for GaAs being similar to an IMPATT. InP has speed and efficiency advantages and should also allow higher peak power and impedance since the peak electric field is limited by the SHG waveform. However, because the field constraint is a waveform effect rather than a property of the device itself, this adds a further feature to the already complex circuit design.

4. Device Geometry and Circuit Properties. - It is easy to appreciate the general principle that low device impedances and short wavelengths imply close tolerance in the circuit elements. Probably the greatest problems relate to the immediate environment of the active layer, ie contacts, metallisation and package design, and it is for these aspects that inferences from the device studies are of most value. The 1-dimensional device analysis relates the voltage and current density for the active layer, a representative large signal impedance for GaAs transferred-electron devices at $100 \mathrm{GHz}$ being $2 \times 10^{-4}$ ohm for $1 \mathrm{~cm}^{2}$. Figures for InP devices and for avalanche diodes are of the same order. Allowing for $Q$ factors up to 10 , contact resistivities below $10^{-5}$ ohm for $1 \mathrm{~cm}^{2}$ are necessary for the resistive loss to be sma1. This is not a too formidable technological requirement in itself.

However, a practical device includes a layer of highly doped semiconductor on at least one side of the active layer with metal contacts either side as sketched in figure 6 . The thickness of this contact layer modifies the acceptable transverse

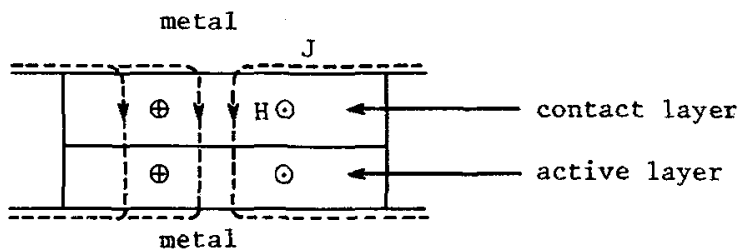

Fig. 6: Device structure. $\mathrm{J}$ indicates current and $\mathrm{H}$ the magnetic field 
dimensions imposed by skin effect considerations. If there were no contact layer, the structure would behave as a transmission line for which the wave slowing factor $n$ would be typically 5. This value of $n$ arises because the dielectric admittance, which would give a slowing factor of $\varepsilon^{\frac{1}{2}}$, is increased by a comparable electronic contribution. For the current to be nearly uniform across the device, its transverse dimensions should be less than about $\lambda / 4 \mathrm{n}$, roughly $150 \mu \mathrm{m}$ at $100 \mathrm{GHz}$. This is not large for a high power device. A contact layer should be thinner than the skin depth so that most of the transverse current flow remains in the metal, but the magnetic field through the contact layer adds to the inductance of the transmission line which increases the slowing factor. For medium and high power devices, the design may need to reduce the contact layer thickness to $\sim 1 \mu \mathrm{m}$. Contact effects are less serious when the active layer thickness is greater, but this condition makes heat dissipation harder. However both the wave slowing effects and heat dissipation favour a geometry with a large ratio of perimeter to cross-sectional area. For this reason, many of the InP transferred-electron oscillators for the 100 to $140 \mathrm{GHz}$ band examined in our laboratory have used a cross geometry.

The circuit beyond the device contacts is constrained by the combination of low device impedance (typically 1 to $10 \mathrm{ohm}$ ) with the need to match both real and imaginary parts in order to set up the required device mode. Good circuit engineering can allow impedance adjustment, but uncontrolled inductance in series with the semiconductor device is hard to compensate. Bond wires in particular are subject to large variations in position with consequential variations of inductance. One approach to this problem is to integrate the semiconductor device with a package including parallel plate electrodes which act as a transformer coupling the device with outside electromagnetic fields. (11) Figure 7 shows an alternative construction, used in RSRE, in which the device is integrated with a silver bridge. This structure bonds naturally into microstrip, coplanar line or finline, in which it acts as a continuation of transmission line up to the plane of the device.

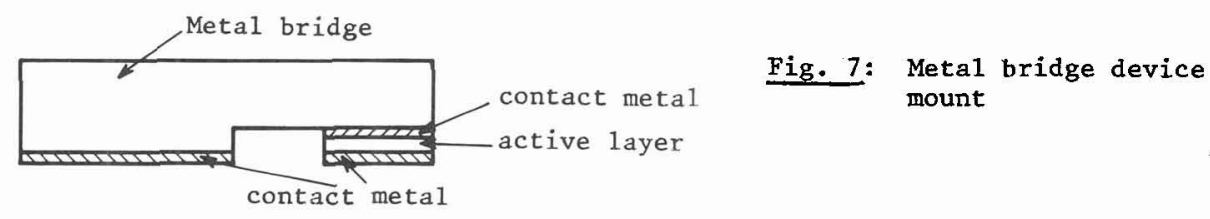

5. Other Hot Electron Sources. - The IMPATT and related diode oscillators operate at very high electric field strengths in their drift regions as well as in carrier generation zones. This is of practical benefit in increasing the power-impedance product and for moderating the slowing factors that restrict the transverse dimensions of devices. However, the high field tends to invalidate the semi-classical picture of the electron motion partly because the field modifies the band states 
themselves and the scattering processes between them and partly because the scattering rates for the energetic electrons are so high that the transitions merge together. Progress is being made towards understanding the microscopic processes (12), but a device analysis is some way off. However some inferences for millimetre band sources can be gathered from experimental data without needing fundamental modelling.

The first general observation is that high field velocity saturation, at about $10^{7} \mathrm{~cm} . \mathrm{s}^{-1}$ or a little less, occurs in all the developed semiconductors except for narrow gap compounds with low avalanche breakdown fields. The similarity of different materials is to be expected since high energy electron and hole distributions will reflect the broad similarities of band structure rather than detailed differences close to turning points. Velocity saturation is readily understood on a semiclassical view, but it must also have a deeper quantum mechanical justification. Its practical significance is that injected carriers drift effectively without diffusion or mutual repulsion at a velocity almost independent of the high frequency electric field, provided of course that the total field is above the saturation threshold and below breakdown. Some of the factors that lead to design complications in vacuum tubes are therefore inherently absent in solid state devices. The offsetting disadvantages are that considerable power dissipation is needed to maintain the carxiers in the velocity saturated condition and that the smallness of the velocity implies transit lengths of about $0.5 \mu \mathrm{m}$ at $100 \mathrm{GHz}$ which in turn 1 imits the device geometry to a parallel plate configuration. Magnetic effects are very weak since momentum relaxation rates are at least $10^{14} \mathrm{~s}^{-1}$, much greater than the accessible cyclotron frequency. This consideration gives some insight into relationships between hot electron solid state and vacuum tube sources. Vacuum tubes use quite complex techniques to drive electrons against the cavity $f i e 1 d$. In solid state devices this is achieved more simply; carrier generation processes replace bunching mechanisms, drift distances are always very short and magnetic deflection is not relevant.

The aspect of high frequency oscillator diodes most influenced by hot electron time delays is the carrier generation process itself. The two accepted generation mechanisms are tunnelling, basically pair production in a very high field region, and avalanche multiplication, in which a high energy primary particle induces the pair production. The former is a strictly quantum phenomenon, usually viewed as instanteous. Avalanche multiplication is subject to a phase delay because the field determines the particle multiplication rate rather than the current itself and this leads to the characteristically higher efficiency of the IMPATT compared with the TUNNETT. The effect of special interest is the extra time for a newly created particle to reach the avalanche threshold energy. Quite significant delays may be inferred from measured avalanche rates. Figure 8 shows the dependence of the measured avalanche factor, $\alpha$, on field for $\mathrm{Si}^{(13)}$, GaAs ${ }^{(14)}$ and InP ${ }^{(15)}$. other 
measurements have been made with different results in detail, but only the rough trend is of present concern.

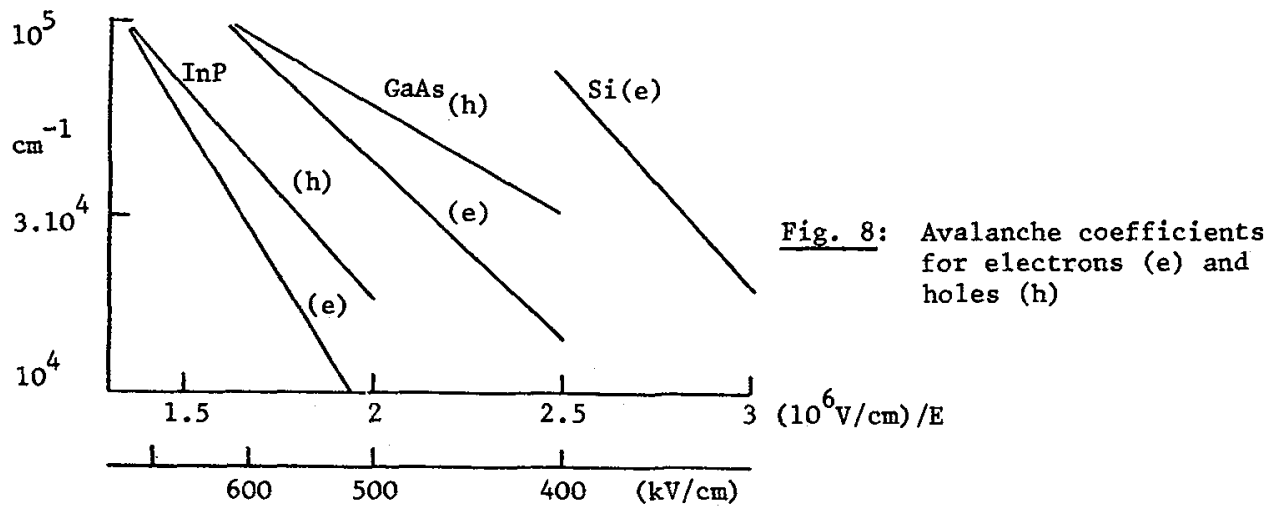

From the $\alpha(E)$ characteristic the time, $T_{a}$, between ionising collisions can be estimated as follows. The distance, $d$, moved by a carrier between its generation and next ionising collision is $k / \alpha$, where $k=\ln (2)$. The energy gained from the field is $\mathrm{kE} / \alpha$ and the energy lost to phonons is $\left(\mathrm{kE} / \alpha-\varepsilon_{\mathrm{a}}\right)$ where $\varepsilon_{\mathrm{a}}$ is the impact ionisation threshold. If the average phonon energy is $\varepsilon_{p}$ and time between scatterings is $\tau$, we see that

$$
T=\left(k E / \alpha-\varepsilon_{a}\right) \tau / \varepsilon_{p}
$$

An alternative expression is $T=k / \bar{v}$, where $\bar{v}$ is the inter-ionisation drift velocity which will be close to the high field drift velocity since fast ballistic-like motion will only occur for a small fraction of the energy gain between ionisations.

The highest field values in figure 8 are quite high for an actual device, so the derived value of $T$ should not be unreasonably long. A representative $\varepsilon_{p}$ is $0.03 \mathrm{eV}$ and, assuming that the accepted electron-phonon scattering rate can be justified for electron energies $1 \mathrm{eV}$ or, so $\tau=5 \times 10^{-15} \mathrm{~s}$ is reasonable. The corresponding estimate of $\mathrm{T}$ is about $0.5 \mathrm{ps}$, which is satisfactorily close to the $\mathrm{ka} / \mathrm{v}$ for $\bar{v}=10^{7} \mathrm{~cm} \cdot \mathrm{s}^{-1}$. Although on the basis of semi-classical theory it is not difficult to obtain a consistent view of the physics, the time between phonon scatterings implies energy uncertainties significantly larger than the phonon energy, so the true interpretation should rest on a quantum model. Since the time for an avalanche to build up will be several times $\mathrm{T}$, it is clear that the avalanche build up will occupy a significant fraction of a cycle at frequencies above $100 \mathrm{GHz}$. The delay will introduce effects somewhat like the differences between the IMPATT and the TUNNETT. There is an overall conclusion that these high frequency diodes will be 
influenced by phase delays that can only be properly assessed by a quantum analysis. One diode of particular practical interest includes a mixed carrier generation process $^{(16)}$ and it is possible that delays in the avalanche build up could favourably compensate the phase advance of the initial pair production by tunnelling.

Recent alternative concepts for millimetre band sources include radiative transsitions between levels in MOS inversion layers at temperatures around $4{ }^{\circ} \mathrm{K}(17,18)$, a derivative of the transferred-electron effect exploiting superlattice states (19) and a cyclotron maser operating with lower energy electrons at reduced temperatures to minimise scattering ${ }^{(20)}$. Only the first of these ideas has been demonstrated experimentally and in this case powers have been very low and obtainable frequencies mainly above the $400 \mathrm{GHz}$ limit set by atmospheric attenuation. However the ideas deserve consideration since device engineering should be able to develop a good principle. The inversion layer structures demonstrate three features absent from existing semiconductor sources, the engineering of the quantum states by a device structure rather than selecting a material, use of 1-electron radiative transitions rather than a co-operative process and direct coupling of the hot electrons to free space "waves without a metallic resonant circuit. For an uncooled millimetre band source, collision broadening will make 1-electron transitions unattractive, but structural engineering of quantum levels might be possible for other devices in which co-operative effects, less sensitive to scattering, are exploited. Direct radiation from hot electrons suggests radical rethinking of circuits, two possible objectives being coupling to structures large or at less comparable in size with the wavelength and the elimination of metal as a high frequency conductor in view of the increasing conduction losses at high frequencies.

The modulation doped superlattice ${ }^{(19)}$ also uses the device structure to engineer the quantum levels, but, in contrast, strong scattering is exploited to give a negative differential resistance. As for the ordinary transferred-electron effect, cooperative instabilities will dominate device characteristics. The anticipated relatively slow transfer rate would not appear to assist high frequency generation, but it remains conceivable that quantum levels could be engineered to produce a bulk-like high frequency oscillator mode. The cyclotron maser ${ }^{(20)}$ is an example of a magnetically-tuned vacuum tube analog. The concept is somewhat at variance with the natural philosophy of solid state devices in that reduced temperatures and low electron energies are needed for the scattering to be tolerably weak and the inherent advantages of velocity saturation and fairly high breakdown fields in semiconductors are ignored. For these reasons, direct analogs of magnetically-tuned tubes seem the least promising avenue for alternative power sources.

6. Schottky Barrier Mixers. - The Schottky barrier diode is the only device so far seriously considered as a mixer for the millimetre band. It is conventionally 
regarded as a cold electron device, but electron heating can occur at high frequencies when the speed of the depletion layer edge exceeds the electron thermal velocity. Under these conditions changes in electrical characteristics become possible and recognition of the physics is essential for device and circuit development. Monte Carlo simulations of the electron motion $n$ GaAs-metal junctions have been used to examine the device physics ${ }^{(21)}$ and a preliminary indication of the high frequency electron heating is provided by figures 9 and 10 . Figure 9 is a sketch of

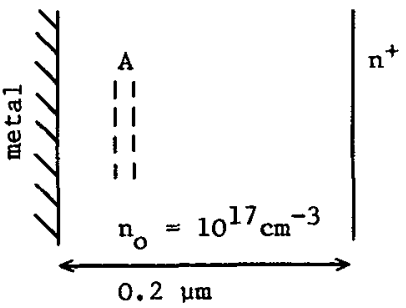

Fig. 9: SB diode. A indicates zone where electron density is plotted in fig.IO.

the simulated structure and figure 10 shows the electron density in the sma11 zone (indicated by $A$ in figure 9) for an applied voltage $v_{0}+v_{1} \cos \omega t$. The values of $v_{0}$ and $v_{1}$ are representative for a mixer. $v_{1}=0.8 \mathrm{~V}$ and $v_{0}$ is defined so that the flat band condition is reached at the maximum of the applied voltage. Curve a pertains to low frequencies and is followed with slight hysterisis up to $200 \mathrm{GHz}$.

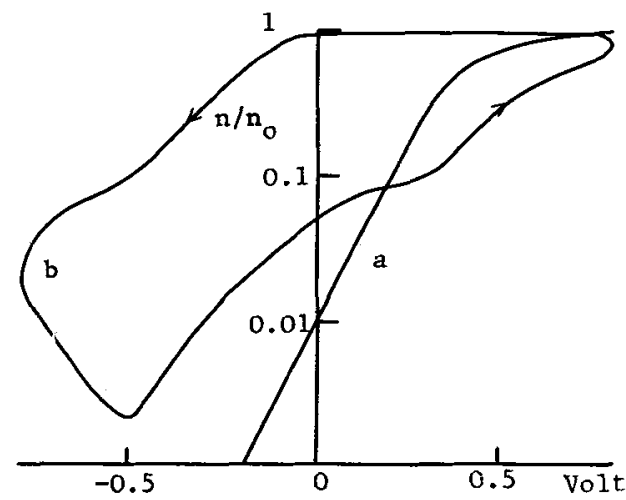

Fig. 10: Electron density variation. a: low frequency b: $800 \mathrm{GHz}$

IOb pertains to $800 \mathrm{GHz}$, the hysterisis becoming stronger and the electron temperature rise to roughly $900^{\circ} \mathrm{K}$ ( 3 times $\mathrm{T}_{\mathrm{o}}$ ) is illustrated by the reduced slope of the $\log (\mathrm{n}) /$ voltage function. The mixer terminal characteristics are being analysed and an interpretation, including doping and bias voltage effects, will be published in due course. 
7. Conclusions. - The emphasis of this paper is consideration of hot electron relaxation effects in 2-terminal devices for millimetre band applications.

3-terminal devices have been proposed as amplifiers for at least part of this band, but have not been considered here since their development is still some way off and electron transport phenomena are even more complex than for 2-terminal sources and mixers. In transferred-electron sources, relaxation effects are significant even at lower frequencies, but the picture is not one of simple degradation with increasing frequency since device modes with fairly favourable properties can be identified and related to experiment. In other devices, both power sources and diodes, relaxation processes become progressively influential at frequencies over $100 \mathrm{GHz}$ and need to be taken into account in the development of the devices themselves and, through impedance effects, on circuit design.

Acknowledgements. - The author is indebted to S J Beard and A M Mazzone for discussions on device theory and for permission to include unpublished material. He is also indebted to $\mathrm{N}$ Apsley and $\mathrm{J}$ E Pattison for discussions on device physics, technology and characteristics.

\section{References}

1. Haydl, W. H., Smith, S. and Bosch, R. : "100 $\mathrm{GHz}$ Gunn diodes fabricated by molecular beam epitaxy", App1. Phys. Lett., 1980, 37, pp 556-7.

2. Crowley, J.D., Sowers, J. J., Janis, B.A. and Fank, F.B.: "High efficiency $90 \mathrm{GHz}$ InP Gunn oscillators", Electron Lett., 1980, 16, pp 705-6.

3. Fawcett, W., Boardman, A.D. and Swain, S.: "Monte Carlo determination of electron transport properties of gallium arsenide", J. Phys. Chem. Solids, 1970,31 , pp 1963-90.

4. Mazzone, A.M. and Rees, H.D.: "Transferred-electron harmonic generators for millimetre band sources", IEE Proc., 1980, 127, Pt. 1, pp 149-60.

5. Ridley, B.K.: "Anatomy of the transferred-electron effect in III-V semiconductors", J. App1. Phys. 1977, 48, pp 754-64.

6. Jones, D. and Rees, H.D.: "Overlength modes of transferred-electron oscillators", Electron. Lett., 1973, 9, pp 105-6.

7. Eastman, L.F. et al: "Ballistic electron motion in GaAs at room temperature", Electron Lett, 1980, 16, pp 524-5.

8. Gray, K.W. et al: "Current limiting contacts for InP transferred-electron devices", Proc. 5th Biennial Cornell Elec. Eng. Conf. 1975, pp 215-24.

9. Chang, K, Sun, C, English, D.L. and Nakaji, E.M.: "High power $94 \mathrm{GHz}$ pulsed IMPATT oscillators", IEEE MTT-S Int Microwave Symposium, 1979, pp 71-2. 
10. Ino, S, Makimura, T. Ishibashi, T. and Ohmori, M: "80 GHz Band High-Power Silicon Diamond Heatsink IMPATT Diodes", Trans Inst. Electron and Comm. Eng. Japan, 1979, E62, pp 712-713.

11. Cachier, G: "Les modules pour ondes millimétriques", Revue Technique ThomsonCSF, 1979, 11, PP 785-822.

12. For a recent discussion see Ferry, D. K: "Modelling of carrier transport in the finite collision duration regime: effects in submicron semiconductor devices", Physics of Nonlinear transport in semiconductors, Plenum Pub. Corp. 1980, pp 577-588.

13. Lee, C.A. et al: "Ionization Rates of Holes and Electrons in Silicon", Phys. Rev. 1964, 134, pp A761-773.

14. Ito, M., Kagawa, S., Kaneda, T, and Yamaoka, T: "Ionization rates for electrons and holes in GaAs", J. Appl. Phys. 1978, 49(8), pp 4607-8.

15. Umebu, I., Choudhury, A.N.M.M. and Robson, P.N. "Ionization coefficients measured in abrupt InP junctions", App1. Phys. Lett. 1980, 36(4), pp 302-3.

16. E1ta, M.E., Fetterman, H.R. Macropoulos, W.V. and Lambert, J.J.: "150 GHz GaAs MTTATT Source", IEEE. ED Lett, 1980, EDL-1, pp 115-6.

17. Gornik, E. and Tsui, D.C.: "Voltage-Tunable Far-Infrared Emission from Si Inversion Layers", Phys. Rev. Lett, 1976, 37, pp 1425-8.

18. Tsui, D.C. and Gornik, E: "Far-infrared emission from Si-MOSFET's on high-index surfaces", Appl. Phys. Lett, 1978 32(6), pp 365-7.

19. Hess, K., Morkoc, H., Shichijo, H, and Streetman, B.G.: "Negative differential resistance through real-space electron transfer", Appl. Phys. Lett., 1979, 35(6), pp 469-71.

20. Ganguly, A.K. and Chu, K.R.: "Theory of a solid-state cyclotron maser", Phys. Rev. B, 1978, 18, pp 6880-9.

2I. Beard, S. J . and Rees, H.D., to be published. 